\title{
Effect of Sperm DNA Fragmentation on Embryo Quality in Normal Responder Women in IVF and ICSI
}

\author{
Dong Suk Kim and Seung-Hun Song \\ Department of Urology, Fertility Center, CHA Gangnam Medical Center, CHA University, Seoul, Korea.
}

To the Editor,

We have read with interest this study by Kim, et al. ${ }^{1}$ regarding the effect of sperm DNA fragmentation (SDF) on embryo quality. Male factor infertility is known to be the sole cause of infertility in $20 \%$ to $30 \%$ of cases of all infertility, and is generally considered to play a role in approximately half of infertile couples. Current diagnostic evaluation of male factor infertility largely depends on the conventional semen analysis, which includes the sperm concentration, motility, and morphology data. However, limitations exist in the diagnosis of male fertility by the conventional semen analysis, and the necessity of more sophisticated diagnostic methods has been continuously suggested even in this period of advanced assisted reproductive technology, such as in vitro fertilization (IVF) or intracytoplasmic sperm injection (ICSI).

Approximately $15 \%$ of patients with male factor infertility were reported to have a normal semen analysis. ${ }^{2}$ There have been many studies investigating the role of sperm DNA integrity in male factor infertility over the past decades. The majority of published data have suggested that the sperm of infertile men contain more DNA damage than fertile men, and that this sperm DNA damage could have a negative effect on fertility potential of these patients. ${ }^{3,4}$ However, the clear impact of sperm DNA damage on the reproductive outcomes still remains controversial. $^{5}$

In this article, Kim et al. have reported that high SDF level resulted in low Day 3 embryo formation rates compared to low SDF level. The authors concluded that SDF level of $<30.7 \%$ could

Received: September 11, 2020 Accepted: September 24, 2020

Corresponding author: Seung-Hun Song, MD, PhD, Department of Urology, Fertility Center, CHA Gangnam Medical Center, CHA University, 566 Nonhyeon-ro, Gangnam-gu, Seoul 06135, Korea.

Tel: 82-2-3468-3413, Fax: 82-2-3468-3449, E-mail: shsong02@cha.ac.kr

-The authors have no potential conflicts of interest to disclose.

(C) Copyright: Yonsei University College of Medicine 2020

This is an Open Access article distributed under the terms of the Creative Commons Attribution Non-Commercial License (https://creativecommons.org/licenses/ by-nc/4.0) which permits unrestricted non-commercial use, distribution, and reproduction in any medium, provided the original work is properly cited. be a good predictor to achieve a top-quality or grade A embryo formation rate of $>70 \%$, in addition to having a potential paternal effect on embryo quality in IVF cycles even in normal responder women. Overall data in the article were generally wellanalyzed, and the cut-off value of SDF ( $>30.7 \%$ ) agrees with the previously reported high SDF cut-off values $(\geq 30 \%)$.

However, a couple of shortcomings and limitations of this study, such its retrospective design with a small sample size which the authors already mentioned in the article, warrant further comments. First of all, the main purpose of SDF test is to evaluate sperm DNA defects in male patients with underlying male factor infertility. In this study, male factor infertility was included as just one cause of infertility in both low and high SDF level groups. We assume that the male factor infertility group would have abnormal semen analysis, and this group should have been analyzed separately from other female factor infertility groups. Another important point is that there is still no clear consensus on the optimal technique or appropriate sperm DNA damage cut-off levels. Until now, various methods for SDF testing assays, such as sperm chromatin structure assay (SCSA) or terminal deoxynucleotidyl transferase-mediated deoxyuridine triphosphate nick end labeling (TUNEL) assay, have been introduced. Among these methods, the sperm chromatin dispersion (SCD) test is known to be a relatively simple, fast, and low-costly option, and therefore, is considered to be more applicable in clinical centers. However, current SDF testing methods, including the Halosperm assay employed in this article, suffer from relatively high inter- and intra-observatory differences.

In summary, we commend the authors and their contribution for demonstrating the potential application of SDF testing in clinical guidance. More subsequent research and large-scale trials are also needed to further substantiate the clinical usefulness of SDF testing in association with reproductive outcomes.

\section{ACKNOWLEDGEMENTS}

This research was supported by the National Research Foun- 
dation of Korea (NRF) grant funded by the Korea government (MSIT) (2018R1C1B508379413).

\section{AUTHOR CONTRIBUTIONS}

Conceptualization: Seung-Hun Song. Data curation: Seung-Hun Song. Formal analysis: Seung-Hun Song. Funding acquisition: SeungHun Song. Methodology: Seung-Hun Song. Project administration: Seung-Hun Song. Resources: Dong Suk Kim and Seung-Hun Song. Supervision: Seung-Hun Song. Visualization: Dong Suk Kim. Writing—original draft: Dong Suk Kim and Seung-Hun Song. Writing-review \& editing: Seung-Hun Song. Approval of final manuscript: all authors.

\section{ORCID iDs}

Dong Suk Kim https://orcid.org/0000-0001-7350-0303

Seung-Hun Song https://orcid.org/0000-0003-4649-9129

\section{REFERENCES}

1. Kim SM, Kim SK, Jee BC, Kim SH. Effect of Sperm DNA fragmen- tation on embryo quality in normal responder women in in vitro fertilization and intracytoplasmic sperm injection. Yonsei Med J 2019;60:461-6.

2. Agarwal A, Allamaneni SS. Sperm DNA damage assessment: a test whose time has come. Fertil Steril 2005;84:850-3.

3. Simon L, Zini A, Dyachenko A, Ciampi A, Carrell DT. A systematic review and meta-analysis to determine the effect of sperm DNA damage on in vitro fertilization and intracytoplasmic sperm injection outcome. Asian J Androl 2017;19:80-90.

4. Borges E Jr, Zanetti BF, Setti AS, Braga DPAF, Provenza RR, Iaconelli A Jr. Sperm DNA fragmentation is correlated with poor embryo development, lower implantation rate, and higher miscarriage rate in reproductive cycles of non-male factor infertility. Fertil Steril 2019;112:483-90.

5. Schulte RT, Ohl DA, Sigman M, Smith GD. Sperm DNA damage in male infertility: etiologies, assays, and outcomes. J Assist Reprod Genet 2010;27:3-12. 